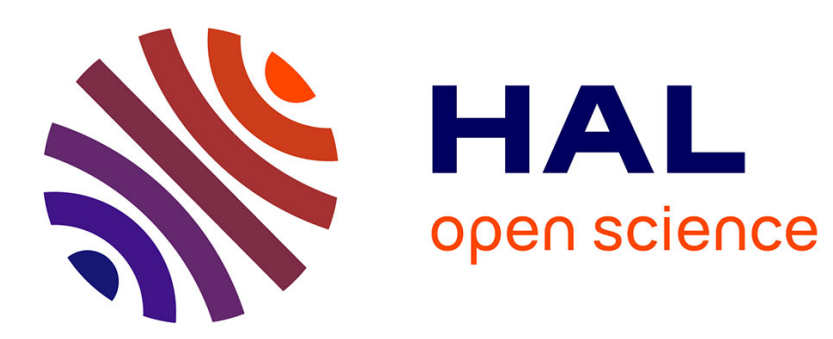

\title{
Adjectif et adjectivité ou comment un substantif peut être plus adjectif qu'un adjectif
}

Mathilde Salles

\section{To cite this version:}

Mathilde Salles. Adjectif et adjectivité ou comment un substantif peut être plus adjectif qu'un adjectif. L'information grammaticale, 2004, 103, pp.7-12. 10.3406/igram.2004.2543 . hal-00012281

\section{HAL Id: hal-00012281 \\ https://hal.science/hal-00012281}

Submitted on 19 Oct 2005

HAL is a multi-disciplinary open access archive for the deposit and dissemination of scientific research documents, whether they are published or not. The documents may come from teaching and research institutions in France or abroad, or from public or private research centers.
L'archive ouverte pluridisciplinaire HAL, est destinée au dépôt et à la diffusion de documents scientifiques de niveau recherche, publiés ou non, émanant des établissements d'enseignement et de recherche français ou étrangers, des laboratoires publics ou privés. 


\section{ADJECTIF ET ADJECTIVITÉ OU COMMENT UN SUBSTANTIF PEUT ÊTRE PLUS ADJECTIF QU'UN ADJECTIF $^{1}$}

Mathilde SALLES

Le constat n'est pas nouveau: la catégorie adjectivale est une catégorie aux contours flous, qui comprend des membres plus ou moins représentatifs, plus ou moins typiques. Ce qui explique sans doute le fait, souligné par Goes (1999: 7), qu'elle ait surtout reçu des définitions négatives. Goes (1999:35) cite à ce propos la conclusion finale de Picabia (1978 : 146) :

Faire l'étude des constructions adjectivales pose le problème fondamental de savoir ce qu'est un adjectif. [...] on ne peut donner que des définitions négatives : un adjectif n'est pas un verbe $[\ldots]$ ce n'est pas une forme participiale ni un substantif.

Puisqu'il est impossible de définir une catégorie floue par un seul critère homogène ou par un ensemble de critères nécessaires et suffisants ${ }^{2}$, Goes, qui cherche à donner une définition positive de cette catégorie grammaticale « tout en tenant compte de ses frontières floues et de ses affinités éventuelles avec d'autres catégories » (p. 34), va donc mettre à l'épreuve les diverses caractéristiques (morphologiques, syntaxiques et sémantiques) généralement attribuées à l'adjectif, afin d'en déterminer les plus saillantes pour la construction d'un prototype abstrait. Aucun des critères utilisés pour définir ce prototype n'est spécifique à l'adjectif (d'autres catégories y répondent), mais, comme le montre Goes en les confrontant à d'autres parties du discours, ils en sont caractéristiques (c'est en quelque sorte à cette catégorie qu'ils s'appliquent «le mieux»). Ces critères sont les suivants :

- la gradation par très,

- en fonction d'épithète, le mouvement de la postposition à l'antéposition et inversement (avec ou sans changement de sens),

- la fonction attribut.

En plus des trois critères syntaxiques utilisés par Goes pour définir le prototype de la catégorie, on peut déterminer une fonction sémantique par excellence de l'adjectif : qualifier des entités, représenter des propriétés d'entités. Là encore, il s'agit d'une représentation privilégiée, mais non exclusive de la catégorie et, là encore, il s'agit d'une propriété qui n'est que typique (certains adjectifs n'y répondent pas, notamment les adjectifs de relation).

Enfin, outre les critères qui définissent le prototype abstrait de la catégorie et qui déterminent le degré d'adjectivité d'une unité, Goes reconnaît deux critères

\footnotetext{
${ }^{1}$ Cet article est une version remaniée et développée d'une section du chapitre «Catégorisation adjectivale et adverbiale » (François, Salles et Lenepveu), dans Catégorisation et langage, Cordier et François (éds), 2002, Paris, Hermès Science Publications, p. 83-105.

${ }^{2}$ Voir Goes (1999 : 35).
} 
nécessaires (mais non suffisants ${ }^{3}$ ) à cette catégorie : l'accord en genre et en nombre avec le support nominal et la fonction d'épithète postposée. Goes propose ainsi une forme d'aménagement de la théorie du prototype - aménagement conforme à ce que préconise Kleiber $(1990)^{4}$ et seul garant du principe de catégorisation - qui consiste à déterminer, à côté des caractéristiques (i.e. les traits typiques) qui forment le prototype abstrait de la catégorie, au moins un trait nécessaire (Goes, on vient de le voir, en détermine finalement deux). Mais on verra plus loin que cette distinction entre deux types de traits ne va pas sans heurts et qu'elle ébranle parfois ce qu'elle est censée consolider, c'est-à-dire la catégorisation.

\section{ADJECTIFS PLUS OU MOINS PROTOTYPIQUES}

De façon générale ${ }^{5}$, les adjectifs qu'on appelle qualificatifs répondent assez bien au prototype de la catégorie, même si certains, tels les adjectifs de couleur ${ }^{6}$, ont du mal à s'antéposer et si d'autres n'acceptent pas ou difficilement le degré. On cite traditionnellement, à ce propos, des adjectifs à valeur superlative comme énorme, gigantesque, minuscule, excellent, exceptionnel - les adjectifs non-classifiants de Milner (1978) - et des adjectifs qui désignent des propriétés non gradables comme mort, carnivore, rectangulaire....

$\mathrm{Au}$ contraire, les adjectifs de relation s'éloignent considérablement du prototype adjectival. Et, à moins d'un transfert dans la catégorie des adjectifs qualificatifs, ils refuseront généralement tous les critères de typicité adjectivale. Dans un discours présidentiel, l'adjectif présidentiel exclut la gradation par très (*un discours très présidentiel), le mouvement de la postposition à l'antéposition (*un

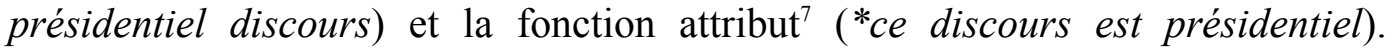
Sémantiquement, ces adjectifs n'attribuent pas une propriété à une entité, mais définissent une relation qu'on peut généralement paraphraser au moyen d'un syntagme prépositionnel : le discours présidentiel est le discours du président, avec ici une relation agentive par rapport au nom discours, i.e. le discours fait par le président.

Ces adjectifs refusent donc généralement les critères qui définissent le prototype abstrait de la catégorie adjectivale, sauf s'il y a un changement dans l'interprétation sémantique de l'adjectif. Un seul exemple, repris à Bally (1944: $\S$ 180): dans le SN une héroïne cornélienne, cornélienne est, selon Bally, susceptible de deux interprétations suivant qu'on parle de Chimène ou de

\footnotetext{
${ }^{3}$ Non suffisants : en effet, les participes passés y répondent qu'ils aient un sens adjectival ou un sens verbal.

${ }^{4}$ Voir Goes (1999 : 41).

${ }^{5}$ Cette généralisation masque évidemment bien des nuances et des différences. Pour une étude précise de ces adjectifs et de leur degré de typicité, voir Goes (1999).

${ }^{6}$ Ils ne s'antéposent que dans des emplois figurés (e.g. de noirs desseins) ou lorsqu'ils sont utilisés comme épithètes de nature (e.g. les vertes prairies, la blanche colombe).

${ }^{7}$ Le refus de la fonction attribut n'est toutefois pas une règle absolue. Bartning (1976) donne plusieurs exemples (parfois attestés) d'adjectifs de relation en fonction attribut et souligne, comme Picabia (1978), que certains emplois contrastifs facilitent la position attribut de ces adjectifs (e.g. cette décision n'est pas présidentielle, mais gouvernementale).
} 
Charlotte Corday. Si l'on parle de Chimène, cornélienne est un adjectif de relation (avec, là encore, une relation agentive : il s'agit d'une héroïne créée par Corneille) qui n'accepte ni la gradation par très, ni l'antéposition, ni la fonction attribut. En revanche, si l'on parle de Charlotte Corday, alors cornélienne ne définit plus une relation, mais attribue une propriété au nom héroïne: il s'agit d'une héroïne qui présente des caractéristiques comparables - l'abnégation, le sens du devoir - à celles des héroïnes cornéliennes (au sens relationnel cette fois). Et alors, certaines des propriétés typiques de l'adjectif deviennent possibles, notamment la gradation par très (une hérö̈ne très cornélienne) et la fonction attribut (cette héroüne est (vraiment) cornélienne). Dans ce type d'emploi, cornélienne prend une valeur qualificative et se rapproche alors du prototype adjectival.

\section{QUELQUES PARADOXES OU COMMENT UN SUBSTANTIF PEUT ÊTRE PLUS ADJECTIF QU'UN ADJECTIF.}

La distinction effectuée par Goes entre deux types de propriétés (les propriétés nécessaires, critères d'intégration dans la classe de l'adjectif, et les propriétés typiques, critères d'adjectivité) se heurte à des paradoxes. En effet, certains éléments, qui sont exclus de la catégorie des adjectifs parce qu'ils ne répondent pas à l'un des deux critères nécessaires, se rapprochent par ailleurs davantage du prototype abstrait que beaucoup d'adjectifs. Par exemple, certains « substantifs adjectivés ${ }^{8}$ (e.g. limite, culte, bateau dans cas limite, film culte, sujet bateau), tout en étant exclus de la classe de l'adjectif parce qu'ils ne répondent pas au critère de l'accord, témoignent d'un plus fort degré d'adjectivité que certains adjectifs, comme les adjectifs de relation (qui, eux, répondent aux deux critères nécessaires, mais ne satisfont généralement aucun des critères typiques).

On peut d'ailleurs se demander ce qui légitime l'intégration de ces derniers dans la catégorie adjectivale, puisque les deux seuls critères auxquels ils répondent (accord en genre et en nombre avec leur support nominal et fonction d'épithète postposée) ne sont pas des critères suffisants. On peut se poser la même question à propos d'autres adjectifs, tels les adjectifs que Gross (1996) appelle typologisants (e.g. accent aigu / grave / circonflexe, enseignement primaire / secondaire / supérieur), qui refusent eux aussi tous les critères de typicité adjectivale, ou encore tels les adjectifs modaux de Milner $(1967)^{9}$ - e.g. simple, ancien, faux, dans un simple / ancien / faux prêtre - qui, eux, en plus d'exclure les critères de typicité adjectivale, ne répondent même pas au critère nécessaire de la fonction d'épithète postposée. Si l'on adopte une perspective homonymique, comme le fait J.-C. Milner, alors les critères de Goes ne permettent plus d'intégrer simple, ancien, faux (dans un simple / ancien / faux prêtre) parmi la catégorie adjectivale. Et c'est généralement lorsqu'on adopte une telle perspective qu'on qualifie les adjectifs de relation, les adjectifs typologisants ou encore les adjectifs modaux de

\footnotetext{
${ }^{8}$ L'expression est utilisée par Goes.

9 Chez Milner (1967), ces adjectifs sont des adjectifs à place fixe, toujours antéposés. Sémantiquement, contrairement à leurs homophones postposés, ils ne définissent pas «l'appartenance à un sous-ensemble, mais une modalité de l'appartenance à l'ensemble » (p. 276). Ils se distinguent encore de leurs homophones postposés par leur comportement syntaxique (ils refusent notamment degré et fonction attribut).
} 
pseudo-adjectifs. Goes, lui, on s'en doute, rejette totalement ce type d'analyse, tout comme il récuse la notion de pseudo-adjectif (cf. p. 253-254). Mais, finalement, une analyse polysémique des unités concernées, si elle sauvegarde le principe de catégorisation adjectivale (de ce point de vue, ancien et secondaire sont des adjectifs qui connaissent des emplois adjectivaux prototypiques $-e$.g. un problème ancien / secondaire - et d'autres peu ou pas prototypiques - e.g. un ancien ministre, l'enseignement secondaire), ne fait que déplacer le problème : certains emplois d'adjectifs ne sont quasiment pas des emplois adjectivaux... et aussi, certains adjectifs (des adjectifs de relation comme présidentiel, cornélienne) connaissent typiquement des emplois peu adjectivaux et exceptionnellement des emplois adjectivaux.

Mais revenons-en aux "substantifs adjectivés ». Le traitement de ces « nouveaux adjectifs » (pour reprendre l'expression plus audacieuse de Noailly 1982) dans les dictionnaires reflète parfois le paradoxe que j'évoquais tout à l'heure. Par exemple, bateau et chic sont tous les deux donnés par le Petit Robert (1990) comme des "adjectifs invariables » dans un sujet bateau, une toilette chic, une réception chic. Et on a là, effectivement, des morphèmes qui présentent un degré d'adjectivité assez fort. Ils autorisent la fonction attribut, et cela sans article :

$$
\begin{aligned}
& \text { a. Ce sujet est bateau. } \\
& \text { b. Cette réception est chic. }
\end{aligned}
$$

Ils permettent aussi la modification par un adverbe de degré :

$$
\begin{aligned}
& \text { a. un sujet très bateau } \\
& \text { b. une réception très chic }
\end{aligned}
$$

Mais ces morphèmes ne répondent qu'à un des deux critères nécessaires de Goes : s'ils autorisent la fonction d'épithète postposée, ils ne s'accordent pas en genre et en nombre avec leur support nominal ${ }^{10}$. En faire des adjectifs invariables, comme le propose le Petit Robert, c'est privilégier les critères typiques auxquels ils répondent (et c'est ainsi privilégier leur fort degré d'adjectivité) aux dépens du critère nécessaire de l'accord. Et c'est d'ailleurs ce que fait Goes lui-même avec chic, puisqu'il lui accorde le statut d'adjectif. Mais alors, à partir de quel moment décide-t-on que l'accord n'est plus un critère décisif pour la catégorisation adjectivale?

En revanche, l'emploi adjectival du substantif culte (film culte, livre culte, auteur culte) n'est mentionné ni par le Petit Robert (1990), ni par Le Grand Robert de la Langue Française (2001). Il s'agit pourtant là d'un emploi bien intégré dans le français contemporain, avec, là aussi, un degré d'adjectivité bien affirmé, comme en témoigne la possibilité de l'utiliser en position d'attribut :

(3) C'est un film qui va devenir culte pour certains.

(Journal de 13 heures, France 2, 11/10/2000)

et d'opérer certaines modifications adverbiales : a. un film vraiment/totalement culte

\footnotetext{
${ }^{10}$ On notera toutefois que l'accord en nombre est de plus en plus souvent fait avec chic.
} 
b. le très culte Citizen Kayne $e^{11}$

Plus étonnant encore, pour ne pas dire plus choquant, est le traitement que réserve le Petit Robert (1990) à choc. Son emploi épithétique n'est pas mentionné sous l'entrée nominale de $c h o c$, mais sous une entrée à part. Une entrée dans laquelle choc est précédé d'un trait d'union (ce que l'usage ne confirme pas nécessairement et ce que ne confirme pas l'exemple de Noailly 1982, tendances chocs) et dans laquelle il ne reçoit aucune catégorisation grammaticale. -Choc y est simplement décrit comme :

[le] deuxième élément de noms composés, signifiant «qui provoque un choc psychologique (surprise, intérêt, émotion...) ». Discours-choc. Mesures-chocs.

L'accord en nombre semble prévu (cf. le deuxième exemple donné par le Petit Robert). On retrouve la même analyse de -choc (toujours précédé de son trait d'union) dans Le Grand Robert de la Langue Française (2001), mais, cette fois, les exemples donnés soulignent son invariabilité :

(5) un discours-choc, des prix-choc, des mesures-choc, une idée choc [sans trait d'union]

invariabilité confirmée par la remarque suivante :

les composés s'écrivent avec ou sans trait d'union, et -choc y est généralement ${ }^{12}$ invariable

Voilà donc -choc perdu dans un no man's land catégoriel: ni adjectif, ni substantif employé adjectivement; sans identité catégorielle et aux propriétés morphologiques incertaines.

Autre reflet de l'embarras provoqué par ce type de substantif: le Petit Robert n'exclut pas l'accord en nombre du substantif épithète limite ${ }^{13}$ (cette fois traité comme un substantif) dans des expériences-limite(s). Goes (p. 152) donne luimême deux exemples attestés avec accord en nombre : les exemples-limites et des situations limites (ce dernier exemple étant repris à Noailly 1982). Et limite n'est, de ce point de vue, ni un cas isolé, ni un cas... limite. Les exemples suivants l'illustrent bien :

(6) amendements surprises, radios pirates, domaines clés, tendances chocs (série d'exemples relevés par Noailly $1982: 131-132$ )

(7) Jadis, ils se fussent coagulés autour de quelques idées-forces ; mais il n'y a plus de marché pour les idées et, à la demande générale, des hommes-forces les ont remplacées. (Paul Morand, Eloge du repos, Arléa, 1996 : 37)

(8) Son effronterie seule grandissait, plus geignarde, plus acharnée, dressée à l'aumône dès le maillot, pareille aux enfants phénomènes qu'on désosse pour les culbutes des cirques. (Emile Zola, La Joie de vivre, Les Rougon-Macquart, vol. 3, La Pléiade, 1981 : 1006)

Noailly (1982 : 132) estime à juste titre que ce type d'accord semble « appelé à se généraliser, signe qu'on traite spontanément ces N2 tels des épithètes »et, pourrait-on ajouter, tels des adjectifs.

${ }^{11}$ Avec en plus ici une position (l'antéposition) exceptionnelle pour un substantif épithète.

${ }^{12} \mathrm{~L}$ 'adverbe généralement cultive le flou.

${ }^{13}$ Substantif qui, lui aussi, autorise la fonction attribut (ce cas est limite) et la spécification par l'adverbe de degré très (un cas très limite). 
On fera une dernière remarque à propos de l'accord : certains adjectifs de couleur, dérivés de substantifs, comme marron, orange, paraissent bien intégrés dans la classe des adjectifs malgré leur invariabilité, espèce de réminiscence morphologique de leur catégorie d'origine. Et, comme le souligne Noailly (1982), en reprenant une remarque de Wagner et Pinchon, les règles d'accord semblent purement arbitraires ici, puisque d'autres adjectifs de couleur, dérivés eux aussi de substantifs, comme mauve, rose, pourpre, s'accordent. L'invariabilité des adjectifs de couleur et des substantifs épithètes de couleur est d'ailleurs mise à mal par nos plus grands auteurs, comme en témoigne la liste d'exemples «non conformes à la règle » donnée par le Bon Usage (13ème édition : § 541), dans laquelle on trouve :

(9) chevaux pies (Hugo); robes rouges, vertes ou oranges (Loti); yeux marrons (Mauriac, Green, Yourcenar) ; lagunes émeraudes (Camus); etc.

Doit-on y voir des erreurs ou des manifestes contre des règles d'accord arbitraires et des catégorisations nominales aberrantes? La deuxième hypothèse est beaucoup plus séduisante.

Si l'accord peut être aussi arbitraire, reste-t-il un critère décisif pour l'intégration dans la classe de l'adjectif? Et qu'est-ce qui prime pour la catégorisation ? Cette propriété morphologique ou le comportement syntaxique et les propriétés sémantiques du morphème ?

Syntaxiquement, on l'a vu, les emplois adjectivaux de limite, bateau, culte répondent aux principales caractéristiques des adjectifs et, ainsi, à la plupart des traits qui définissent le prototype adjectival. Sémantiquement, ces " substantifs » qualifient, attribuent une propriété ${ }^{14}$ et prennent souvent une valeur sémantique propre, distincte des valeurs sémantiques liées aux emplois nominaux. Et, avec cette valeur sémantique propre, le passage à la fonction attribut se fera de préférence, sinon toujours, sans déterminant :

(10) ce sujet est bateau $v s^{*}$ ce sujet est un bateau

(11) ce projet est bidon $v s *$ ce projet est un bidon $v s$ ? ce projet est du bidon

(12) ce cas est limite $v s$ ? ce cas est une limite

(13) ce film est culte $v s$ ? ce film est un culte

A la suite de Victorri (2002: 112), on soulignera tout de même des différences parmi ces sens adjectivaux des substantifs épithètes : alors que limite et culte dans un cas limite, un film culte "conserve[nt] presque intégralement [leur] sens nominal $»^{15}$, bateau a, quant à lui, un sens adjectival nettement séparé des sens nominaux et bidon un sens adjectival nettement séparé des sens nominaux

\footnotetext{
${ }^{14}$ Ce qui n'est pas le cas de tous les substantifs épithètes. Certains, comme ceux que Noailly (1990) appelle substantifs épithètes de complémentation (la stratégie Mitterrand, le vote Mitterrand, un réparateur télé) introduisent une relation sémantique avec le nom recteur comparable à celle des adjectifs de relation (respectivement agent, bénéficiaire et objet, dans les exemples donnés). Mais ceux que Noailly nomme substantifs épithètes de qualification (un cas limite, un sujet bateau, un film culte, une couleur tendance) ont d'évidentes affinités avec les adjectifs qualificatifs.

${ }^{15}$ Victorri fait cette remarque pour limite.
} 
comptables, ce qui explique les non sens produits par *ce sujet est un bateau, ${ }^{*} c e$ projet est un bidon ${ }^{16}$. Mais, malgré ces différences, aucun de ces « substantifs » ne retrouve, dans cette acception, ses droits de substantif, contrairement à des substantifs épithètes de qualification comme événement, monument dans un livre événement, un film monument, qui, eux, retrouveront leurs droits de substantifs et un article propre, en fonction d'attribut :

(14) ce livre est un événement $v s *$ ce livre est événement

(15) ce film est un monument $v s *$ ce film est monument

L'emploi adjectival de tendance illustre parfaitement cette dérive sémantique vers une valeur propre et proprement adjectivale. Aujourd'hui, cet emploi adjectival connaît un grand succès, du moins chez certaines catégories de locuteurs (la presse féminine, par exemple, en est particulièrement friande). Ainsi, on entend fréquemment parler d'une couleur ou d'une marque tendance, très tendance, d'un vêtement qui est vraiment tendance, etc., avec, on peut le souligner, un fort degré d'adjectivité, marqué par la possibilité d'être modifié par l'adverbe de degré très et d'apparaître en fonction attribut sans déterminant. Cet emploi adjectival de tendance (synonyme de branché ou encore de mode, autre substantif aux emplois adjectivaux productifs) a pris une telle ampleur qu'il est même à l'origine d'un nouvel emploi nominal, dans lequel tendance désigne non plus une orientation parmi d'autres, mais la qualité de ce qui est tendance, c'est-à-dire à la mode :

(16) Toujours chic, Chanel attaque la tendance avec des pistolets dorés et strassés à porter à la ceinture ou en broche. (ELLE, n²904, 27 août 2001)

(17) Depuis quelques semaines, «Le Monde Télévision » dégringole vers des zones de lubricité fangeuse. Est-ce opportunisme, avant-gardisme, «tendance $»^{17}(\ldots)$ ? (courrier des lecteurs du Monde Télévision, 15-16 avril 2001)

On assiste, en outre, à une dérive progressive de ce sens nominal vers un autre sens, métonymique, dans lequel tendance désigne l'objet tendance :

(18) Pas cher le look people. Bonne nouvelle pour les chasseuses de tendances : même les must have « vus sur les stars » sont soldés. (ELLE, $\mathrm{n}^{\circ} 2975,6$ janvier 2003)

(19) D'ailleurs, chaque saison, mon placard regorge de tendances. (ELLE, $\mathrm{n}^{\circ} 2884,9$ avril 2001)

La nouvelle valeur sémantique de tendance, liée à son emploi adjectival, donne ainsi lieu à de nouvelles valeurs nominales, qui, si leur emploi se généralisait, pourraient être considérées comme un second phénomène de conversion.

\section{DES CARACTÉRISTIQUES MORPHOLOGIQUES ADJECTIVALES}

Des substantifs épithètes de qualification comme limite, culte, tendance, etc. présentent donc des traits syntaxiques et sémantiques qui les rapprochent indiscutablement des adjectifs. Ils présentent aussi des propriétés qui les éloignent des substantifs. Certains d'entre eux, on l'a constaté avec les exemples (10)-(11) et, dans une moindre mesure, avec les exemples (12)-(13), perdent en effet une

\footnotetext{
${ }^{16}$ Non sens qu'on ne retrouve pas avec l'article partitif (? ce projet est du bidon; ce projet, c'est du bidon), qui sélectionne une autre acception du substantif bidon.

17 André Meunier me signale (c.p.) que les guillemets constituent sans doute la marque d'un emploi non intégré. Il s'agit probablement en même temps d'une marque de polyphonie; l'auteur de la lettre reprend ainsi une expression à la mode sans pour autant se l'approprier.
} 
propriété distributionnelle spécifique aux substantifs : la compatibilité avec un déterminant, et ainsi, finalement, l'aptitude à former un syntagme nominal. Mais, d'un point de vue morphologique, ils conservent apparemment - mis à part quelques accords occasionnels - leurs propriétés nominales.

Cela signifie-t-il qu'ils soient dénués de toute caractéristique morphologique adjectivale ? Certains phénomènes dérivationnels tendent à prouver que non. En effet, parmi les substantifs épithètes de qualification évoqués jusqu'ici, quelquesuns se prêtent à des créations lexicales par suffixation purement adjectivales. Par exemple, culte, tendance (et peut-être limite), peuvent servir de base à des adjectifs en -issime :

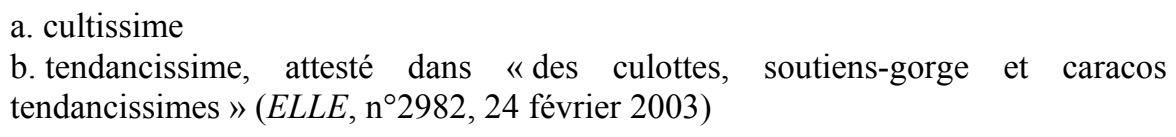

Or, les adjectifs en -issime sont typiquement des adjectifs déadjectivaux.

Vis-à-vis de cette suffixation, culte et tendance adoptent un comportement nettement adjectival. Ils s'opposent en cela aux adjectifs de relation. Il n'est pas inutile d'évoquer ici - pour opposer une fois encore les propriétés très adjectivales de certains substantifs épithètes de qualification aux propriétés peu adjectivales des adjectifs de relation - les remarques de Apothéloz (2002: 65) sur le comportement des adjectifs de relation face à l'affixation :

une propriété relativement constante de ces adjectifs est que, lorsqu'ils sont soumis à une affixation, celle-ci a systématiquement pour résultat sémantique de composer l'affixe avec le nom que cet adjectif transpose. Ainsi, (il) fiscalise est mieux traduit par «soumet à un impôt, soumet au fisc », que par «agit de façon à rendre fiscal»; parlementarisme peut être paraphrasé comme «attitude ou système politique consistant à favoriser l'existence d'un parlement (fort)» (et par extension « régime comportant un parlement »), plutôt que comme « attitude ou système politique de type parlementaire ».

Et Apothéloz conclut en ces termes :

en dépit de leur morphosyntaxe adjectivale, les adjectifs de relation se comportent vis-à-vis de l'affixation, comme d'ailleurs de beaucoup d'autres opérations, comme des noms (on peut d'ailleurs définir les adjectifs de relation comme des noms à morphosyntaxe adjectivale) (Apothéloz $2002: 65$; c'est moi qui souligne)

On n'observe rien de tel avec cultissime, tendancissime: la valeur intensive du suffixe -issime s'applique bien à la valeur sémantique adjectivale de culte, tendance (et non à une valeur nominale) et, vis-à-vis de cette suffixation en issime, culte et tendance se comportent bien comme des adjectifs.

Un autre phénomène, beaucoup plus régulier celui-ci, souligne une affinité d'ordre morphologique entre certains substantifs dits adjectivés et les adjectifs. Beaucoup d'adjectifs donnent lieu, par conversion (ou " dérivation impropre »), à une création nominale. Ce type de conversion est systématique avec les adjectifs de couleur; à partir de chacun d'eux, on a formé un substantif de couleur masculin (bleu $\rightarrow$ le bleu, rouge $\rightarrow$ le rouge, gris $\rightarrow$ le gris, etc.). La conversion 
est double ou, plus exactement, on a affaire à deux opérations de conversion successives (cf. Kerleroux 1996: 152 sq., sur les trois orange), lorsque ces adjectifs sont eux-mêmes issus de substantifs. Ainsi, à partir du nom féminin orange, on a créé l'adjectif de couleur orange, puis le nom de couleur, masculin, orange. Même chose pour marron, mauve, rose :

$$
\begin{aligned}
& \text { a. subst. le marron } \rightarrow \text { adj. de couleur marron } \rightarrow \text { subst. de couleur le marron } \\
& \text { b. subst. la mauve } \rightarrow \text { adj. de couleur mauve } \rightarrow \text { subst. de couleur le mauve } \\
& \text { c. subst. la rose } \rightarrow \text { adj. de couleur rose } \rightarrow \text { subst. de couleur le rose }
\end{aligned}
$$

avec un passage systématique au masculin pour la conversion nominale, quel que soit le genre du substantif d'origine. Les substantifs adjectivés de couleur, dès lors que leur emploi se généralise, n'échappent pas à cette règle de conversion : à partir de l'emploi adjectival de crème ou turquoise, on a formé les substantifs de couleur le crème, le turquoise, avec là aussi un passage au masculin, indépendamment $\mathrm{du}$ genre du substantif d'origine (féminin pour crème et turquoise).

Ces régularités de conversion s'expliquent mal lorsqu'on adopte les distinctions catégorielles traditionnelles, avec rouge, bleu, rose, mauve, marron, adjectifs de couleur (variables ou non, issus d'un substantif ou non), d'un côté, et crème, turquoise, substantifs adjectivés de couleur, de l'autre. Elles semblent plaider, au contraire, en faveur d'un traitement uniforme de tous ces termes de couleur. Cela dit, si les seuls substantifs adjectivés concernés sont ceux qui désignent des couleurs, la portée d'un tel argument est assez limitée. J'ai évoqué plus haut un nouvel emploi nominal de tendance, issu de son emploi adjectival, mais ce nouvel emploi n'hérite pas, contrairement aux noms de couleur, du genre masculin caractéristique de ce type de conversion: tendance reste un nom féminin. Le passage au masculin, pour exprimer la « qualité de ce qui est tendance » se fera-til un jour? On pourrait se poser la même question à propos d'un substantif (masculin) qui désignerait la « qualité de ce qui est limite»?

\section{CONCLUSION}

Parmi les substantifs épithètes, ceux qui, comme limite, tendance, culte, bateau, présentent un fort degré d'adjectivité ne sont pas les plus nombreux. A l'intérieur même de la catégorie des "substantifs épithètes de qualification » de Noailly (1990), beaucoup s'accommodent mal, sinon pas du tout, de la fonction attribut sans déterminant, de la modification par très et presque aucun d'entre eux n'accepte l'antéposition. Une telle résistance aux propriétés adjectivales conduit à traiter la plupart d'entre eux comme des cas de simple distorsion catégorielle et non comme des cas de conversion morphologique (je reprends cette distinction à Kerleroux 1996). Distorsion catégorielle, c'est-à-dire distorsion de nature syntaxique qui ne modifie pas l'appartenance catégorielle du morphème. Ainsi caméléon, kangourou, kleenex, dans un président caméléon, une hausse kangourou, l'amour kleenex ${ }^{18}$, sont bien des substantifs dans un emploi adjectival. Exclusivement épithètes postposées, ils n'héritent d'aucun des traits adjectivaux prototypiques évoqués par Goes et ils conservent leur intégrité morphologique

\footnotetext{
18 Tous ces exemples sont repris à Noailly (1990).
} 
nominale. Il paraît donc exclu d'y voir des cas de conversion morphologique. Au contraire, lorsqu'il y a, comme dans le cas de limite, tendance, culte, bateau, un héritage presque complet ${ }^{19}$ des traits adjectivaux prototypiques, il semble difficile de parler d'une simple distorsion catégorielle. Ce serait masquer les différences importantes qui existent entre les emplois adjectivaux de kleenex, caméléon, etc. et ceux de limite, tendance, etc., qui, on l'a vu, ont non seulement une souplesse syntaxique très adjectivale, mais aussi, quelquefois, des caractéristiques morphologiques adjectivales. L'opération d'adjectivation ne laisse donc pas leur intégrité nominale intacte (cf. aussi les exemples (10) à (13)). Malgré le blocage de l'accord (selon la norme, beaucoup plus conservatrice que l'usage), on peut être tenté de leur accorder un statut d'adjectif à part entière, autrement dit d'y voir de véritables conversions morphologiques.

\section{Mathilde SALLES Université de Caen, CRISCO - UMR 6170}

\section{Références bibliographiques}

APOTHeloz D. (2002), La construction du lexique français, Gap-Paris, Ophrys.

BALLY Ch. (1944), Linguistique générale et linguistique française, $4^{\mathrm{ème}}$ édition, Berne, Francke.

BARTNING I. (1976), Remarques sur la syntaxe et la sémantique des pseudo-adjectifs dénominaux en français, Acta Universitatis Stockholmiensis n 10 , Stockholm.

Goes J. (1999), L'adjectif. Entre nom et verbe, Paris, Bruxelles, Duculot.

GRAND ROBERT DE LA LANGUE FRANÇAISE (2001), deuxième édition, dirigée par A. Rey, du Dictionnaire alphabétique et analogique de la langue française de Paul Robert.

Grevisse M. (1993), Le Bon Usage (13ème édition, refondue par A. Goosse), Paris-Louvain-laNeuve, Duculot.

Gross G. (1996), Les expressions figées en français, Gap-Paris, Ophrys.

KERLEROUX F. (1996), La coupure invisible. Etudes de syntaxe et de morphologie, Lille, P.U. du Septentrion.

KLEIBER G. (1990), La sémantique du prototype, Paris, PUF.

MiLnER J.-C. (1967), « Esquisse à propos d'une classe limitée d'adjectifs en français moderne », M.I.T. Quaterly Progress Report, 84, Research Laboratory of Electronic, p. 275-285.

MiLner J.-C. (1978), De la syntaxe à l'interprétation. Quantités, insultes, exclamations, Paris, Le Seuil.

NOAILlY M. (1982), « De nouveaux adjectifs », Le français moderne, vol. 50, n², p. 129-139.

NOAILly M. (1990), Le substantif épithète, Paris, P.U.F.

PETIT ROBERT (1990), dictionnaire alphabétique analogique de la langue française, rédaction dirigée par A. Rey et J. Rey-Debove, Paris, Le Robert.

PICABIA L. (1978), Les constructions adjectivales en français, Genève, Droz.

VictorRi, B. (2002), «La catégorisation et la polysémie », in F. Cordier et J. François (éds), Catégorisation et langage, Paris, Hermès Science Publications, p. 107-124.

${ }^{19}$ Seule l'antéposition reste extrêmement rare. 